\section{Failure of Supplementary Ultraviolet Radiation to Enhance Flower Color under Greenhouse Conditions}

\author{
Richard M. Klein \\ Botany Department, University of Vermont, Burlington, VT 05405 \\ Additional index words. UV-A, UV-B, anthocyanin content of flower petals
}

\begin{abstract}
In order to determine whether the concentration of floral petal anthocyanin pigments could be increased, ultraviolet radiations in the UV-A and UV-B wavelength bands were presented to a variety of flowering plants to partly restore those wavelengths filtered out by greenhouse glass. In no tested plant did the supplementary ultraviolet radiation enhance floral anthocyanin content. Supplementary UV radiation has no economic value in greenhouse production of flowering plants.
\end{abstract}

A factor in the market acceptability of flowering annual plants sold for bedding or as gift plants is the brilliance of their flowers. Since, in New England, such plants are frequently grown under glass, the radiation environment must be considered in commercial production. Phytochrome and/or the high-energy blue-absorbing pigments play roles in the induction of enzyme systems leading to the synthesis of blue and red anthocyanin pigments (Mohr and Drumm-Herrel, 1983). The participation of ultraviolet wavelengths in anthocyanin synthesis has been known for more than a century (Bonner, 1880; Klein, 1976). For several test systems, either the near UV (UV-A $=320$ to $385 \mathrm{~nm}$ ) or the middle UV (UV-B $=290$ to $320 \mathrm{~nm}$ ) or both activate one or more of the enzymes involved in anthocyanin synthesis (Beggs and Wellmann, 1985).

Although greenhouse glass is transparent to the visible and most UV-A wavelengths, UV-B is completely filtered out (Klein, 1979). Since both UV-A and UV-B may affect synthesis of floral anthocyanins, addition of these wavelengths might make it possible to grow flowering plants with more brilliant flowers. A study was conducted in which greenhouse solar radiation was supplemented with UVA and UV-B radiation to determine if supplementary UV radiation might have some economic value in commercial production of flowering plants.

Luminaires consisting of one each of commercially available UV-A lamps (BLB fluorescent lamps with emission peaking at 365 $\mathrm{nm}$ ) and UV-B lamps (Westinghouse FS Sunlight lamps filtered through two layers of

Received for publication 13 Mar. 1989. Research supported by the Vermont Agricultural Experiment Station. The assistance of the laboratories of Martyn M. Caldwell (Utah State Univ.) and Alan Teramura (Univ. of Maryland) in providing spectral energy distribution measurements is gratefully acknowledged. The cost of publishing this paper was defrayed in part by the payment of page charges. Under postal regulations, this paper therefore must be hereby marked advertisement solely to indicate this fact.

${ }^{1}$ Professor of Botany.
Kodacel to remove wavelengths below 285 $\mathrm{nm})$ were used as sources of supplementary UV radiation. The spectral energy distribution curves of unfiltered solar UV in the greenhouse, of solar UV filtered through greenhouse glass, of the UV-enhanced spectrum, and of the spectral energy distribution minaire. of the luminaire are presented in Fig. 1. The supplementary UV radiation had a slightly lower energy flux than present in solar radiation in the 300 to $320 \mathrm{~nm}$ range, although it should be adequate to saturate those pigments that control anthocyanin synthesis (Reinert et al., 1964; Mohr and DrummHerrel, 1983).

With the exception of African violet (Saintpaulia), three geranium (Pelargonium) cultivars, and hyacinth (Hyacinths) bulbs, all plants were grown from commercially available seed. Test plants were grown in a Cornell potting soil mix (Bunt, 1976). Plants were watered daily and received $20 \mathrm{~N}-20 \mathrm{P}$ $20 \mathrm{~K}$ soluble fertilizer once a week. Supplementary visible radiation was not provided. Four or more plants of each cultivar or species were used. Control plants received only solar radiation filtered by the greenhouse glass. Plants exposed to supplementary UV radiation were grown under ordinary greenhouse regimes until apparently mature, but before there was a visible indication of flower stalks or flower primordia. At this time, which varied with the species, plants were placed under the supplementary UV luminaires and

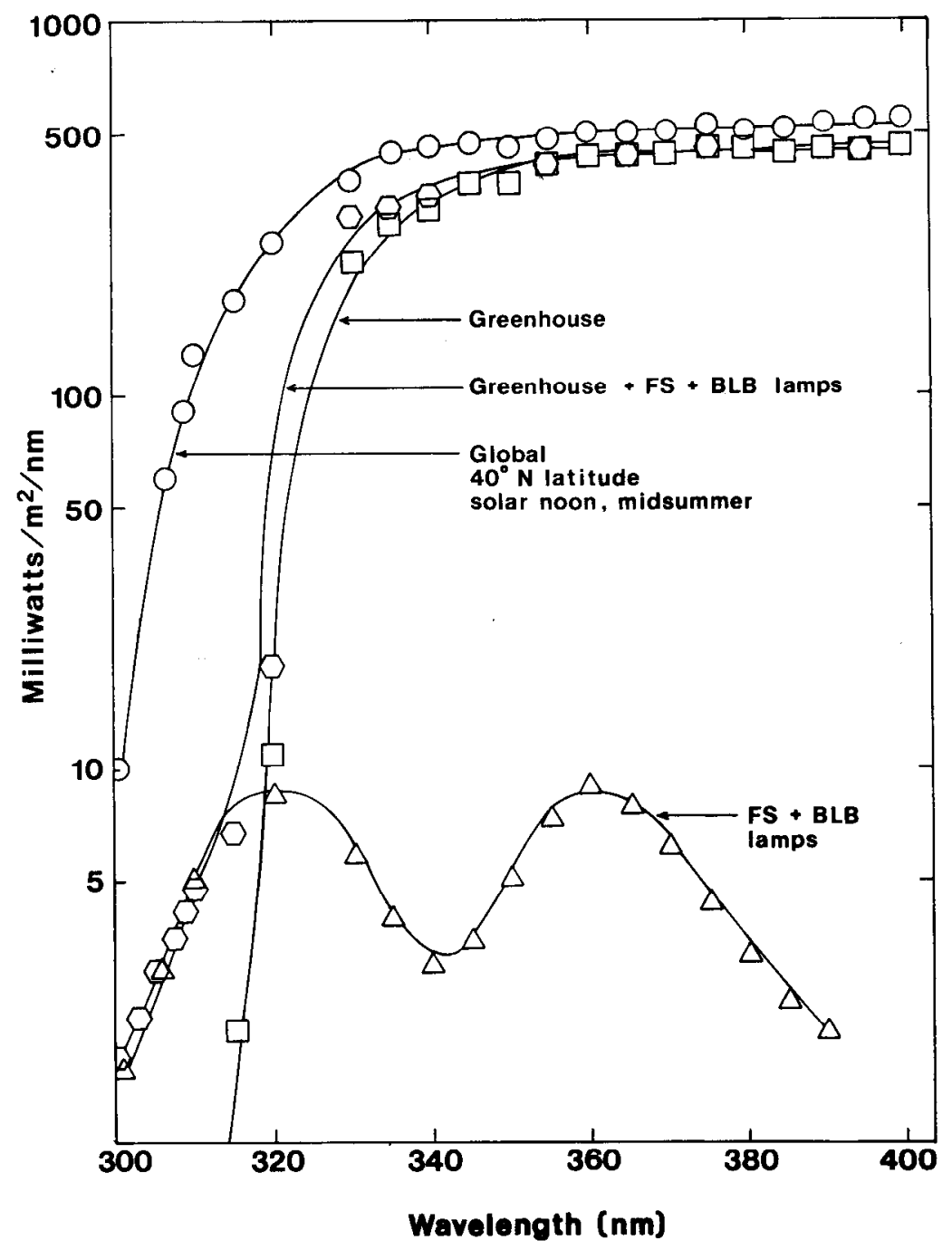

Fig. 1. Spectral energy distribution curves of the ultraviolet spectra of solar radiation under greenhouse glass, solar radiation filtered through greenhouse glass, emission from a luminaire of UV-B and UVA fluorescent lamps, and of glass-filtered solar radiation amended with radiation from the UV lu- 
Table 1. Absorption of $535 \mathrm{~nm} \pm$ SD of anthocyanins extracted with $5 \mathrm{ml}$ of 97 methanol : $3 \mathrm{HCl}$ $(\mathrm{v} / \mathrm{v})$ from individual, whole, mature flowers grown under greenhouse conditions with sunlight filtered through greenhouse glass with or without supplementary UV-A and UV-B radiation from fluorescent lamps.

\begin{tabular}{|c|c|c|c|c|c|}
\hline \multirow[b]{2}{*}{ Cultivar } & \multirow[b]{2}{*}{ Source $^{2}$} & \multirow[b]{2}{*}{ Dilution } & \multicolumn{3}{|c|}{ Absorption $(535 \mathrm{~nm})$} \\
\hline & & & Control & & $+\mathrm{UV}$ \\
\hline \multicolumn{6}{|c|}{ Geranium (Pelargonium $\times$ hortorum $(n=15)$} \\
\hline Applause & $\mathrm{GH}$ & $1: 1$ & $0.44 \pm 0.06$ & 0.36 & \pm 0.06 \\
\hline Strawberry Blossom & P $3583-7$ & 0 & $0.033 \pm 0.007$ & 0.038 & \pm 0.00 \\
\hline Pink Double & $\mathrm{GH}$ & $1: 1$ & $0.35 \pm 0.07$ & 0.32 & \pm 0.07 \\
\hline Pink Edge & $\mathrm{GH}$ & 0 & $0.18 \pm 0.05$ & 0.19 & \pm 0.07 \\
\hline Red Elite & $\mathrm{GH}$ & $1: 2$ & $0.95 \pm 0.04$ & 0.95 & \pm 0.10 \\
\hline Orbit Deep Salmon & P 1511-4 & $1: 2$ & $0.69 \pm 0.05$ & 0.82 & \pm 0.12 \\
\hline Orbit Salmon & P $1515-2$ & $1: 1$ & $0.67 \pm 0.08$ & 0.67 & \pm 0.08 \\
\hline Orbit Cardinal & P $1506-3$ & $1: 1$ & $0.41 \pm 0.05$ & 0.39 & \pm 0.04 \\
\hline \multicolumn{6}{|c|}{ Pansy $($ Viola $\times$ wittrockiana Gams $)(n=25)$} \\
\hline White & P $1475-6$ & 0 & $0.04 \pm 0.02$ & 0.05 & \pm 0.02 \\
\hline True Blue & P $1465-5$ & $1: 10$ & $0.14 \pm 0.05$ & 0.13 & \pm 0.04 \\
\hline Deep Blue & P $1460-5$ & $1: 10$ & $0.20 \pm 0.07$ & 0.23 & \pm 0.07 \\
\hline Yellow & P $1482-1$ & 0 & $0.59 \pm 0.22$ & 0.64 & \pm 0.21 \\
\hline Orange & P $1473-2$ & $1: 2$ & $0.15 \pm 0.05$ & 0.20 & \pm 0.05 \\
\hline Rose & P $1461-7$ & $1: 3$ & $0.47 \pm 0.12$ & 0.54 & \pm 0.24 \\
\hline Scarlet & P $1472-0$ & $1: 10$ & $0.78 \pm 0.17$ & 0.66 & \pm 0.15 \\
\hline \multicolumn{6}{|c|}{ Phlox (Phlox drummondi Hook.) $(n=20)$} \\
\hline White Beauty & B 47076 & 0 & $0.02 \pm 0.01$ & 0.02 & \pm 0.01 \\
\hline Blue Beauty & B 47076 & $1: 1$ & $0.69 \pm 0.23$ & 0.69 & \pm 0.30 \\
\hline Pink Beauty & B 47076 & $1: 1$ & $0.13 \pm 0.04$ & 0.14 & \pm 0.05 \\
\hline \multicolumn{6}{|c|}{ Impatiens (Impatiens balsamina L.) $(n=25)$} \\
\hline Gem Rose Pink & P $3502-7$ & 0 & $0.60 \pm 0.14$ & 0.56 & \pm 0.16 \\
\hline Gem Rose & P 3498-6 & $1: 2$ & $0.79 \pm 0.13$ & 0.79 & \pm 0.15 \\
\hline Gem Violet & P 3503-9 & $1: 1$ & $0.75 \pm 0.12$ & 0.76 & \pm 0.28 \\
\hline Gem Red & P $3499-8$ & $1: 4$ & $0.51 \pm 0.07$ & 0.50 & \pm 0.08 \\
\hline \multicolumn{6}{|c|}{ Lobelia (Lobelia erinus L) $(n=25)$} \\
\hline Cambridge Blue & TM 1633 & $1: 1$ & $0.06 \pm 0.01$ & 0.06 & \pm 0.02 \\
\hline Crystal Palace & TM 1638 & $1: 1$ & $0.21 \pm 0.06$ & 0.15 & \pm 0.05 \\
\hline Red Cascade & TM 1640 & $1: 1$ & $0.21 \pm 0.05$ & 0.28 & \pm 0.06 \\
\hline Rosamund & TM 6468 & $1: 1$ & $0.27 \pm 0.05$ & 0.24 & \pm 0.04 \\
\hline \multicolumn{6}{|c|}{ Petunia (Petunia $\times$ hybrida) $(n=25)$} \\
\hline Ultra Pink & P $1641-9$ & $1: 1$ & $0.22 \pm 0.05$ & 0.22 & \pm 0.04 \\
\hline Ultra Rose & P $1635-6$ & $1: 2$ & $0.57 \pm 0.16$ & 0.53 & \pm 0.15 \\
\hline Ultra Red & P $1642-1$ & $1: 10$ & $0.44 \pm 0.08$ & 0.35 & \pm 0.03 \\
\hline Ultra Blue & P $1637-0$ & $1: 10$ & $0.69 \pm 0.11$ & 0.60 & \pm 0.14 \\
\hline Ultra Burgundy & P $1632-0$ & $1: 10$ & $0.67 \pm 0.11$ & 0.65 & \pm 0.07 \\
\hline \multicolumn{6}{|c|}{ Periwinkle (Vinca rosea L.) $(n=25)$} \\
\hline Little Pinky & B 46615 & $1: 1$ & $0.54 \pm 0.24$ & 0.50 & \pm 0.18 \\
\hline Little Bright Eye & B 46615 & $1: 1$ & $0.05 \pm 0.02$ & 0.06 & \pm 0.02 \\
\hline \multicolumn{6}{|c|}{ Stock [Matthiola incana (L.) R. Br] $(n=20)$} \\
\hline Trisomic White & P $1933-8$ & 0 & $0.05 \pm 0.02$ & 0.08 & \pm 0.03 \\
\hline Trisomic Pink & P $1933-8$ & $1: 10$ & $0.05 \pm 0.02$ & 0.03 & \pm 0.01 \\
\hline Trismonic Purple & P $1933-8$ & $1: 10$ & $0.26 \pm 0.12$ & 0.24 & \pm 0.16 \\
\hline \multicolumn{6}{|c|}{ Salvia (Salvia splendens F. Sellow ex Roem. \& Schutt) $(n=15)$} \\
\hline Blaze of Fire & TM 1840 & $1: 15$ & $0.50 \pm 0.14$ & 0.57 & \pm 0.14 \\
\hline Claryssa Pink & TM 2040 & $1: 1$ & $0.51 \pm 0.16$ & 0.49 & \pm 0.18 \\
\hline Farin. Victoria & TM 1817 & $1: 5$ & $0.40 \pm 0.09$ & 0.36 & \pm 0.08 \\
\hline Farin. Blue Bed & TM 1816 & $1: 5$ & $0.41 \pm 0.06$ & 0.36 & \pm 0.06 \\
\hline \multicolumn{6}{|c|}{ Hyacinth (Hyacinthus orientalis $L)(n=10)$} \\
\hline Blue Delft & $\mathrm{AG}$ & $1: 3$ & $0.58 \pm 0.14$ & 0.60 & \pm 0.08 \\
\hline Blue Jacket & $\mathrm{AG}$ & $1: 3$ & $0.83 \pm 0.16$ & 0.81 & \pm 0.33 \\
\hline Pink Pearl & $\mathrm{AG}$ & $1: 3$ & $0.27 \pm 0.14$ & 0.26 & \pm 0.13 \\
\hline Violet Pearl & AG & $1: 3$ & $0.45 \pm 0.22$ & 0.41 & \pm 0.20 \\
\hline \multicolumn{6}{|c|}{ African violet (Saintpaulia ionantha) $(n=20)$} \\
\hline Pink Crusader & F 474 & $1: 10$ & $0.26 \pm 0.05$ & 0.14 & \pm 0.06 \\
\hline Kingwood Red & F 338 & $1: 50$ & $0.29 \pm 0.06$ & 0.26 & \pm 0.09 \\
\hline Kingwood Blue & F 579 & 1:5 & $0.17 \pm 0.05$ & 0.20 & \pm 0.06 \\
\hline Huron Sky Blue & F 579 & $1: 5$ & $0.23 \pm 0.06$ & 0.24 & \pm 0.05 \\
\hline
\end{tabular}

${ }^{2}$ Sources: GM = UVM greenhouse; P = Park Seed Co., Greenwood, S.C.; B = W. Atlee Burpee Co., Warminster, Pa.; TM = Thompson and Morgan Co., Jackson, N.J.; AG = Agway Farm Store, Essex Junction, Vt.; F = Fischer Greenhouses, Linwood, N.J. so positioned at a right angle to the solar arc that they received the same exposure to solar illumination as did the controls. The photoperiod of the UV lamps was adjusted to match that of the week. Fully expanded individual flowers that had, as determined in preliminary experiments, attained full size and pigmentation were collected, pigmented flower stalks were removed, and the flowers placed in $5 \mathrm{ml}$ of 97 methanol : $3 \mathrm{HCl}(\mathrm{v} / \mathrm{v})$. Anthocyanin was extracted at $4 \mathrm{C}$ in darkness for 1 to 4 days and anthocyanin determined as absorbance at $535 \mathrm{~nm}$ in a Spectronic 21 spectrophotometer (B \& L). Where necessary, extracts were diluted with methanol$\mathrm{HCl}$ as noted in Table 1.

Supplementary illumination with UV-A and UV-B had no effect on anthocyanin production in the corolla of flowers of a variety of plants at the UV fluxes provided (Table 1). The UV radiation had no visible effect on the appearance or the vegetative or reproductive growth of test plants, although higher UV irradiances are known to damage plants (Hashimoto and Tijima, 1980; Semeniuk, 1982). A requirement for UV-B radiation in anthocyanin synthesis, although apparently demonstrated for native alpine plants (Schanz, 1919), does not seem to exist for these cultivated plants. There may be no UV requirement for anthocyanin synthesis in these species or, over time, any such requirement may have been selected against. There is no economic benefit in providing supplementary UV radiation in production of flowering plants.

\section{Literature Cited}

Beggs, C.J. and E. Wellmann. 1985. Analysis of light-controlled anthocyanin formation in coleoptiles of Zea mays L.: The role of UV-B, blue, red and far red light. Photochem. Photobiol. 41:481-486.

Bonner, C. 1880. De la variation avec l'altitude des matières colorés des fleurs chez une même espéce végetale. Bul. Soc. Bot. France 27:103105.

Bunt, A.C. 1976. Modern potting composts. Pennsylvania State Univ. Press, University Park.

Hashimoto, T. and M. Tajima. 1980. Effect of ultraviolet radiation on growth and pigmentation in seedlings. Plant Cell Physiol. 21:15591577.

Klein, R.M. 1976. Plants and near-ultraviolet radiation. Bot. Rev. 44:1-127.

Klein, R.M. 1979. Cut-off filters for the near ultraviolet. Photochem. Photobiol. 29:1052-1054.

Mohr, H. and H. Drumm-Herrel. 1983. Coaction between phytochrome and blue/UV light in anthocyanin synthesis in seedlings. Physiol. Plant. 58:408-414.

Reinert, J.H., H. Clauss, and R. von Ardenne. 1964. Anthocyaninbildung vom Happlopappus gracilis in Licht von Verschiedener Qualität. Naturwissenschaften 51:87-96.

Semeniuk, F. 1982. Comparative sensitivity of florist and nursery plants to increased UV-B radiation. HortScience 17:519. (Abstr.)

Schanz, F. 1919. Wirkung des Lichtes verschiedener Wellenlänge auf die Pflanzen. Ber. Deutsch. Bot. Ges. 37:430-442. 